\title{
REACHABILITY OF NONNEGATIVE EQUILIBRIUM STATES FOR THE SEMILINEAR VIBRATING STRING BY VARYING ITS AXIAL LOAD AND THE GAIN OF DAMPING*
}

\author{
Alexander Y. Khapalov ${ }^{1}$
}

\begin{abstract}
We show that the set of nonnegative equilibrium-like states, namely, like $\left(y_{d}, 0\right)$ of the semilinear vibrating string that can be reached from any non-zero initial state $\left(y_{0}, y_{1}\right) \in H_{0}^{1}(0,1) \times$ $L^{2}(0,1)$, by varying its axial load and the gain of damping, is dense in the "nonnegative" part of the subspace $L^{2}(0,1) \times\{0\}$ of $L^{2}(0,1) \times H^{-1}(0,1)$. Our main results deal with nonlinear terms which admit at most the linear growth at infinity in $y$ and satisfy certain restriction on their total impact on $(0, \infty)$ with respect to the time-variable.
\end{abstract}

Mathematics Subject Classification. 93, 35.

Received March 31, 2004. Revised December 9, 2004.

\section{INTRODUCTION}

\subsection{Problem setting}

In this paper we consider the following initial and boundary-value problem for the semilinear one dimensional wave equation:

$$
\begin{gathered}
y_{t t}=y_{x x}+v(x, t) y-\gamma(t) y_{t}-F(x, t, y), \quad x \in(0,1), t>0, \\
y(0, t)=y(1, t)=0, \quad y(x, 0)=y_{0}(x), \quad y_{t}(x, 0)=y_{1}(x) .
\end{gathered}
$$

In (S) the functions $v \in L^{\infty}((0,1) \times(0, \infty))$ and $\gamma \in L^{\infty}(0, \infty)$ are regarded as bilinear or multiplicative controls (which we "can choose"). The nonlinear term $F$ is assumed to be fixed but not necessarily known. We regard it as a "disturbance" from a certain class of functions whose properties are described below. Our goal is to investigate the reachability (or controllability) properties of model (S). Namely:

Given any non-zero initial state $\left(y_{0}, y_{1}\right) \in H_{0}^{1}(0,1) \times L^{2}(0,1)$, we would like to know what states $\left(y(\cdot, t), y_{t}(\cdot, t)\right)$ can be reached by system $(S)$ at times $t>0$ by applying various aforementioned bilinear controls $v$ and $\gamma$.

Remark 1.1. One can easily notice that, if $F(x, t, 0)=0$, then the zero state $\left(y_{0}, y_{1}\right)=(0,0)$ is a fixed point for the solution mapping of system $(\mathrm{S})$, regardless of the choice of controls $v$ and $\gamma$. Hence, it cannot be steered anywhere from this state.

Keywords and phrases. Semilinear wave equation, approximate controllability, multiplicative controls, axial load, damping.

* This work was supported in part by NSF Grant DMS-0204037.

1 Department of Mathematics, Washington State University, Pullman, WA 99164-3113, USA; khapala@math.wsu.edu 
If (S) models the oscillations of vibrating string with clapped ends, then $v(x, t)$ can be regarded as an axial load at point $x$ and time $t$ (having in mind that $v$ changes the natural frequency response of the string), and $\gamma(t)$ as the gain of viscous (motion-activated) damping, acting upon the string at time $t$. More generally, a reachability problem like (S) may arise in the context of so-called "smart materials", whose properties can be altered by applying various external factors, such as temperature, electrical current, or magnetic field.

To our knowledge, the problem of bilinear controllability was addressed for the first time in [4] for the linear version of system like (S) and with no damping present, that is, with $F=0, \gamma=0$, namely, as follows:

$$
\begin{gathered}
w_{t t}=w_{x x}+v w, \quad x \in(0,1), \quad t>0 \\
w(0, t)=w(1, t)=0, \quad w(x, 0)=w_{0}(x), \quad w_{t}(x, 0)=w_{1}(x) .
\end{gathered}
$$

Assuming that all the modes in the initial datum are active:

$$
\left(\int_{0}^{1} w_{0} \sin \pi k x \mathrm{~d} x\right)^{2}+\left(\int_{0}^{1} w_{1} \sin \pi k x \mathrm{~d} x\right)^{2} \neq 0 \quad \forall k=1, \ldots
$$

it was shown in [4] that the set of all states which system $\left(\mathrm{S}^{*}\right)$ can achieve at times $t>1$, making use of controls $v \in L^{r}(0, \infty), r \geq 1$, is dense in $H_{0}^{1}(0,1) \times L^{2}(0,1)$.

The methods of [4], based on the non-harmonic analysis and dealing with the Riesz bases of exponential time-functions, are not of explicit nature and do not immediately apply to a semilinear problem like $S$. (More references are provided in the end of this section.)

\subsection{Main result}

In this paper we investigate the reachability/controllability properties of the semilinear system (S) from an arbitrary non-zero initial state. Our methods are quite different from those of the aforementioned work [4] and allow us to employ explicit bilinear controls $v$ and $\gamma$. On the other hand, our main results deal only with the steering properties of system (S) to, what we call, "non-negative target states of equilibrium type" (or just "non-negative equilibrium states") like

$$
\left(y_{d}, 0\right), \quad y_{d}(x) \geq 0 \text { a.e. in }(0,1) .
$$

Remark 1.2. To justify this name, let us note first of all that the actual equilibrium states of (S) (if such exists) look like (1.1a). It can also be shown (see, e.g., [12]) that the set of equilibrium states of truncated version of (S) with $F=0$ and $\gamma=0$, namely, such that

$$
y_{d x x}+\bar{v}(x) y_{d}=0, \quad x \in(0,1)
$$

for some $\bar{v} \in L^{\infty}(0,1)$, is dense in $H_{0}^{1}(0,1)$.

Our main results deal with the following classes functions $v, \gamma$, and $F$ in (S).

Structural and regularity assumptions on $v, \gamma$, and $F$ :

$$
\begin{gathered}
v=v_{*}(x)+h(t), \quad v_{*} \in L^{\infty}(0,1), \quad v_{*}(x) \leq \mu<0 \text { a.e. in }(0,1) ; \\
h(t)= \begin{cases}h_{1} & \text { for } t \in\left(0, t_{1}\right), \\
h_{2} & \text { for } t>t_{1},\end{cases}
\end{gathered}
$$


where $\mu<0, h_{1}<0, t_{1}>0$, and $h_{2}>0$ are some real numbers;

$$
\gamma(t)= \begin{cases}0 & \text { for } t \in\left(0, t_{1}\right) \\ \gamma & \text { for } t>t_{1}\end{cases}
$$

where $\gamma$ is some positive number.

Throughout the paper we assume that $F(x, t, u)$ is measurable in $x, t \in(0,1) \times(0, T)$ for all $T>0$, continuous in $u \in R$ and

$$
|F(x, t, u)| \leq G(t)(1+|u|) \text { for a.a. } x, t \in(0,1) \times(0, \infty), \forall u \in R,
$$

where $G \in L^{\infty}(0, \infty) \bigcap L^{1}(0, \infty)$ is some positive-valued function.

We use below (1.4a) in a different form, namely, we assume that $G$ can be represented as

$$
G(t)=q(t) g(t), \quad g(t), q(t)>0 \text { for a.a. } t \in(0, \infty)
$$

(for example, $q=g=G^{1 / 2}$ ) and

$$
q, g \in L^{\infty}(0, \infty) \bigcap L^{2}(0, \infty)
$$

In other words, we assume that $F$ admits the following representation:

$$
F(x, t, y(x, t))=q(t) f(x, t, y(x, t)) \quad\left(f(x, t, y(x, t))=\frac{F(x, t, y(x, t))}{q(t)}\right)
$$

where

$$
|f(x, t, u)| \leq g(t)(1+|u|) \text { for a.a. } x, t \in(0,1) \times(0, \infty), \forall u \in R
$$

Denote:

$$
\|g\|_{L^{\infty}(0, \infty)}=K,\|g\|_{L^{2}(0, \infty)}^{2}=L,\|q\|_{L^{\infty}(0, \infty)}=B, \quad\|q\|_{L^{2}(0, \infty)}^{2}=D .
$$

Conditions (1.2 a-b)-(1.5) ensure that system $(\mathrm{S})$ has a unique solution $y \in C\left([0, T] ; H_{0}^{1}(0,1)\right) \bigcap C^{1}([0, T]$; $\left.L^{2}(0,1)\right)$ for every pair $\left(y_{0}, y_{1}\right) \in H_{0}^{1}(0,1) \times L^{2}(0,1)$ and every $T>0$.

Remark 1.3. Denote by $\lambda_{k}$ and $\omega_{k}, k=1, \ldots$ respectively the eigenvalues and the orthonormal in $L^{2}(0,1)$ eigenfunctions associated with the spectral problem

$$
\omega_{x x}+v_{*}(x) \omega=\lambda \omega, \quad \omega \in H_{0}^{1}(0,1) .
$$

Then (1.2a) implies that

$$
0>\lambda_{1}>\lambda_{2}>\ldots
$$

Our main result is as follows.

Theorem 1.1. Let (1.2a-b)-(1.5) hold. Consider an arbitrary non-zero initial state $\left(y_{0}, y_{1}\right) \in H_{0}^{1}(0,1) \times$ $L^{2}(0,1),\left(y_{0}, y_{1}\right) \neq(0,0)$, and any non-negative target equilibrium-like state $\left(y_{d}, 0\right), y_{d}(x) \geq 0, y_{d} \in L^{2}(0,1)$. Then for every $\varepsilon>0$ there are a time $\bar{T}=T\left(\varepsilon, y_{0}, y_{1}, y_{d}, L, K, B, D\right)>0$ and piecewise constant-in-time controls $(v, \gamma)$ of the form (1.2a-b)-(1.3) such that

$$
\left\|y_{d}-y(\cdot, T)\right\|_{L^{2}(0,1)}+\left\|y_{t}(\cdot, T)\right\|_{H^{-1}(0,1)} \leq \varepsilon .
$$

(In the above $H^{-1}(0,1)=\left(H_{0}^{1}(0,1)\right)^{\prime}$.) 
As the reader noticed, in Theorem 1.1 we require the initial datum in (S) be of a higher regularity compared to that of the target state. This "discrepancy" arises within the framework of our method due to the presence of nonlinear term $F$ in $(\mathrm{S})$.

Our approach to prove Theorem 1.1 is to try first to find bilinear controls $v$ and $\gamma$ of the form (1.2a-b)-(1.3), which steer the homogeneous truncated version of $(\mathrm{S})$, namely,

$$
\begin{gathered}
u_{t t}=u_{x x}+v(x, t) u-\gamma(t) u_{t}, \quad x \in(0,1), \quad t>0, \\
u(0, t)=u(1, t)=0, \quad u(x, 0)=y_{0}(x), \quad u_{t}(x, 0)=y_{1}(x),
\end{gathered}
$$

from the same initial state $\left(y_{0}, y_{1}\right)$ toward the same target $\left(y_{d}, 0\right)$. While doing this, we intend to select the aforementioned pair $(v, \gamma)$ in such a way that they would generate a trajectory of the original system $(\mathrm{S})$ that follows "closely" to that of (1.8). We will use the following auxiliary result.

Theorem 1.2. Consider an arbitrary non-zero initial state $\left(y_{0}, y_{1}\right) \in H_{0}^{1}(0,1) \times L^{2}(0,1)$ and any non-negative target equilibrium-like state $\left(y_{d}, 0\right), y_{d}(x) \geq 0, y_{d} \in L^{2}(0,1)$. Then for every $\varepsilon>0$ there are $\overline{a \text { time } T=}$ $T\left(\varepsilon, y_{0}, y_{1}, y_{d}\right)>0$ and piecewise constant-in-time controls $(v, \gamma)$ of the form (1.2a-b)-(1.3) such that

$$
\left\|y_{d}-u(\cdot, T)\right\|_{L^{2}(0,1)}+\left\|u_{t}(\cdot, T)\right\|_{H^{-1}(0,1)} \leq \varepsilon
$$

As a matter of fact, in the recent work [12] we already established the controllability result of Theorem 1.2 in the stronger energy space $H_{0}^{1}(0,1) \times L^{2}(0,1)$, which yields $(1.9)$, even for the case of not-necessarily non-negative equilibrium target states. (In [13] we obtained a similar result by making use of a single, more complex, bilinear control $v$.) However, the methods of $[12,13]$ do not directly apply to the semilinear version of (S). In particular, in $[12,13]$ the corresponding controllability results were achieved by applying "relatively small" (i.e., "more practical") multiplicative controls for "relatively long" periods of time.

In the present paper such approach will not work due to the presence of the nonlinear term, because the latter would "corrupt" the dynamics of the system at hand over a "long" period of time. Therefore, making use of the qualitative ideas of [12], in the present paper we will try to achieve the desirable controllability results by applying "relatively large" controls either for "very short" or for suitably selected "large" periods of time, which will allow us to overcome the presence of the additive nonlinear term. However, this will result in the change to a weaker space $L^{2}(0,1) \times H^{-1}(0,1)$ in $(1.7)$.

In this paper we give a new, quite different proof of Theorem 1.2, compared to [12], specifically tailored as an auxiliary step to prove Theorem 1.1 (to ensure, e.g., (4.14), (4.15) in the proof below).

Remark 1.4 (more references on bilinear controllability).

- In [14] the methods of [4] were applied in the context of simultaneous control of the rod equation and Schrödinger equation.

- In recent works [7-11] several global approximate controllability results were obtained for rather general semilinear parabolic equation with nonlinear terms admitting superlinear growth.

- Aside from bilinear controllability, a very close issue is stabilization by means of bilinear controls. We can point out only at a very limited number of publications in this area in terms of pde's, see $[2,3,6,18]$.

- Regarding the issue of optimal control for bilinear systems, we refer to [5,15-17] and the references therein.

- An extensive and thorough bibliography on controllability on bilinear ode's is available, see, e.g., the survey in [1]. 


\subsection{Some useful observations and notations}

Assuming that (1.2a) holds, introduce the following norm equivalent to the standard one in $H_{0}^{1}(0,1)$ :

$$
\|\phi\|_{v_{*}}=\left(\sum_{k=1}^{\infty}\left(-\lambda_{k}\right)\left(\int_{0}^{1} \phi \omega_{k} \mathrm{~d} x\right)^{2}\right)^{1 / 2}=\left(\int_{0}^{1}\left(\phi_{x}^{2}-v_{*}(x) \phi^{2}\right) \mathrm{d} x\right)^{1 / 2} .
$$

Accordingly, we will use the following norm in $H^{-1}(0,1)$ dual of $H_{0}^{1}(0,1)$ :

$$
\|\phi\|_{H^{-1}(0,1)}=\left(\sum_{k=1}^{\infty}\left(-\lambda_{k}\right)^{-1}\left(\int_{0}^{1} \phi \omega_{k} \mathrm{~d} x\right)^{2}\right)^{1 / 2} .
$$

We introduce next the following energy norm:

$$
E^{1 / 2}(\phi(\cdot, t))=\left(\frac{1}{2}\|\phi\|_{v_{*}}^{2}+\frac{1}{2}\|\phi\|_{L^{2}(0,1)}^{2}\right)^{1 / 2}
$$

Note that (1.7) deals with a weaker energy norm, namely, as follows:

$$
E_{*}^{1 / 2}(\phi(\cdot, t))=\left(\frac{1}{2}\|\phi\|_{L^{2}(0,1)}^{2}+\frac{1}{2}\|\phi\|_{H^{-1}(0,1)}^{2}\right)^{1 / 2} .
$$

\section{Solution Formulas For (S)}

Consider any $T>0,\left(y_{0}, y_{1}\right) \in H_{0}^{1}(0,1) \times L^{2}(0,1)$ and any $v, \gamma, q$, and $f$ satisfying conditions $(1.2 \mathrm{a}-\mathrm{b})-(1.5)$. Denote

$$
p(x, t)=F(x, t, y(x, t))=q(t) f(x, t, y(x, t)) .
$$

Note that, due to (1.4)-(1.5), we have $p \in L^{2}\left(Q_{t}\right)$ and for any $t>0$ :

$$
\begin{gathered}
\iint_{Q_{t}} p^{2} \mathrm{~d} x \mathrm{~d} t \leq B^{2} \iint f^{2} \mathrm{~d} x \mathrm{~d} t \leq 2 L B^{2}\left(1+\max _{\tau \in[0, t]}\left\{\int_{0}^{1} y^{2}(x, \tau) \mathrm{d} x\right\}\right) \\
\iint_{Q_{t}} p^{2} \mathrm{~d} x \mathrm{~d} t \leq B^{2} \iint_{Q_{t}} f^{2} \mathrm{~d} x \mathrm{~d} t \leq 2 K^{2} B^{2} t\left(1+\max _{\tau \in[0, t]}\left\{\int_{0}^{1} y^{2}(x, \tau) \mathrm{d} x\right\}\right), \\
\sup _{\tau \in(0, t)} \int_{0}^{1} p^{2} \mathrm{~d} x=\sup _{\tau \in(0, t)} \int_{0}^{1} q^{2}(\tau) f^{2}(x, \tau, y(x, \tau)) \mathrm{d} x \leq 2 K^{2} B^{2}\left(1+\max _{\tau \in[0, t]}\left\{\int_{0}^{1} y^{2}(x, \tau) \mathrm{d} x\right\}\right) .
\end{gathered}
$$

Consider any $v(x)=v_{*}(x)+h$, where $v_{*}$ is as in $(1.2 \mathrm{a})$ and $h$ is any real number. Then the eigenvalues and the orthonormalized (in $\left.L^{2}(0,1)\right)$ eigenfunctions, associated with the spectral problem

$$
\omega_{x x}+v(x) \omega=\lambda \omega, \quad \omega \in H_{0}^{1}(0,1)
$$

are respectively $\lambda_{1}+h>\lambda_{2}+h>\ldots$ and $\omega_{1}, \omega_{2}, \ldots$ (see $\left.(1.6 \mathrm{a}-\mathrm{b})\right)$. 
We have the following classical formula for solutions to the boundary problem (S) with this $v(x)$ :

$$
y(x, t)=\sum_{k=1}^{\infty} c_{k}(t) \omega_{k}(x), \quad x \in(0,1), \quad t>0
$$

where

$$
\begin{gathered}
\frac{\mathrm{d}^{2} c_{k}}{\mathrm{~d} t^{2}}=\left(\lambda_{k}+h\right) c_{k}-\gamma \frac{\mathrm{d} c_{k}}{\mathrm{~d} t}+\int_{0}^{1}(-p(x, t)) \omega_{k}(x) \mathrm{d} x, \quad t>0, \\
c_{k}(0)=\int_{0}^{1} y_{0} \omega_{k} \mathrm{~d} x, \quad \dot{c}_{k}(0)=\int_{0}^{1} y_{1} \omega_{k} \mathrm{~d} x .
\end{gathered}
$$

Solving the system of ordinary differential equations (2.3a-b) yields:

For the index $k$ such that $\gamma^{2}+4\left(\lambda_{k}+h\right)=0$ :

$$
c_{k}(t)=\mathrm{e}^{-\frac{\gamma}{2} t} c_{k}(0)+t\left(\dot{c}_{k}(0)+\frac{c_{k}(0) \gamma}{2}\right) \mathrm{e}^{-\frac{\gamma}{2} t}+\int_{0}^{t} \mathrm{e}^{\gamma(t-\tau) / 2}(t-\tau) \int_{0}^{1}(-p(x, \tau)) \omega_{k}(x) \mathrm{d} x .
$$

For all $k$ 's such that $\gamma^{2}+4\left(\lambda_{k}+h\right)>0$ :

$$
\begin{gathered}
c_{k}(t)=\mathrm{e}^{\left(-\frac{\gamma}{2}+\sqrt{\gamma^{2} / 4+\lambda_{k}+h} t\right.} \frac{\dot{c}_{k}(0)-c_{k}(0)\left(-\frac{\gamma}{2}-\sqrt{\gamma^{2} / 4+\lambda_{k}+h}\right)}{\sqrt{\gamma^{2}+4\left(\lambda_{k}+h\right)}} \\
+\mathrm{e}^{\left(-\frac{\gamma}{2}-\sqrt{\gamma^{2} / 4+\lambda_{k}+h} t\right)} \frac{-\dot{c}_{k}(0)+c_{k}(0)\left(-\frac{\gamma}{2}+\sqrt{\gamma^{2} / 4+\lambda_{k}+h}\right)}{\sqrt{\gamma^{2}+4\left(\lambda_{k}+h\right)}}+\int_{0}^{t}\left[\mathrm{e}^{\left(-\frac{\gamma}{2}+\sqrt{\gamma^{2} / 4+\lambda_{k}+h}(t-\tau)\right.} \frac{1}{\sqrt{\gamma^{2}+4\left(\lambda_{k}+h\right)}}\right. \\
\left.-\mathrm{e}^{\left(-\frac{\gamma}{2}-\sqrt{\gamma^{2} / 4+\lambda_{k}+h}\right)(t-\tau)} \frac{1}{\sqrt{\gamma^{2}+4\left(\lambda_{k}+h\right)}}\right] \int_{0}^{1}(-p(x, \tau)) \omega_{k}(x) \mathrm{d} x \mathrm{~d} \tau ; \quad(2.3 \mathrm{~d}) \\
\dot{c}_{k}(t)=\mathrm{e}^{\left(-\frac{\gamma}{2}+\sqrt{\gamma^{2} / 4+\lambda_{k}+h}\right) t}\left(-\frac{\gamma}{2}+\sqrt{\gamma^{2} / 4+\lambda_{k}+h}\right) \frac{\dot{c}_{k}(0)-c_{k}(0)\left(-\frac{\gamma}{2}-\sqrt{\gamma^{2} / 4+\lambda_{k}+h}\right)}{\sqrt{\gamma^{2}+4\left(\lambda_{k}+h\right)}} \\
+\mathrm{e}^{\left(-\frac{\gamma}{2}-\sqrt{\gamma^{2} / 4+\lambda_{k}+h}\right) t}\left(-\frac{\gamma}{2}-\sqrt{\gamma^{2} / 4+\lambda_{k}+h}\right) \frac{-\dot{c}_{k}(0)+c_{k}(0)\left(-\frac{\gamma}{2}+\sqrt{\gamma^{2} / 4+\lambda_{k}+h}\right)}{\sqrt{\gamma^{2}+4\left(\lambda_{k}+h\right)}} \\
\quad+\int_{0}^{t}\left[\left(-\frac{\gamma}{2}+\sqrt{\gamma^{2} / 4+\lambda_{k}+h}\right) \mathrm{e}^{\left(-\frac{\gamma}{2}+\sqrt{\gamma^{2} / 4+\lambda_{k}+h}\right)(t-\tau)} \frac{1}{\sqrt{\gamma^{2}+4\left(\lambda_{k}+h\right)}}\right. \\
\left.+\left(\frac{\gamma}{2}+\sqrt{\gamma^{2} / 4+\lambda_{k}+h}\right) \mathrm{e}^{\left(-\frac{\gamma}{2}-\sqrt{\gamma^{2} / 4+\lambda_{k}+h}\right)(t-\tau)} \frac{1}{\sqrt{\gamma^{2}+4\left(\lambda_{k}+h\right)}}\right] \int_{0}^{1}(-p(x, \tau)) \omega_{k}(x) \mathrm{d} x \mathrm{~d} \tau . \quad(2.3 \mathrm{e})
\end{gathered}
$$


For all $k$ 's such that $\gamma^{2}+4\left(\lambda_{k}+h\right)<0$ :

$$
\begin{array}{r}
c_{k}(t)=c_{k}(0) \mathrm{e}^{-\frac{\gamma}{2} t} \cos \left(t \sqrt{-\gamma^{2} / 4-\lambda_{k}-h}\right)+\mathrm{e}^{-\frac{\gamma}{2} t} \sin \left(t \sqrt{-\gamma^{2} / 4-\lambda_{k}-h}\right) \frac{\dot{c}_{k}(0)+\gamma c_{k}(0) / 2}{\sqrt{-\gamma^{2} / 4-\lambda_{k}-h}} \\
+\int_{0}^{t}\left[\mathrm{e}^{-\frac{\gamma(t-\tau)}{2}} \frac{\sin \left((t-\tau) \sqrt{-\gamma^{2} / 4-\lambda_{k}-h}\right)}{\sqrt{-\gamma^{2} / 4-\lambda_{k}-h}}\right] \int_{0}^{1}(-p(x, \tau)) \omega_{k}(x) \mathrm{d} x
\end{array}
$$

$$
\begin{gathered}
\dot{c}_{k}(t)=-c_{k}(0) \mathrm{e}^{-\frac{\gamma}{2} t} \sqrt{-\gamma^{2} / 4-\lambda_{k}-h} \sin \left(t \sqrt{-\gamma^{2} / 4-\lambda_{k}-h}\right) \\
+\frac{-\gamma}{2} \mathrm{e}^{-\frac{\gamma}{2} t} \sin \left(t \sqrt{-\gamma^{2} / 4-\lambda_{k}-h}\right) \frac{\dot{c}_{k}(0)+\gamma c_{k}(0) / 2}{\sqrt{-\gamma^{2} / 4-\lambda_{k}-h}} \\
+\mathrm{e}^{-\frac{\gamma}{2} t} \dot{c}_{k}(0) \cos \left(t \sqrt{-\gamma^{2} / 4-\lambda_{k}-h}\right)+\int_{0}^{t}\left[-\frac{\gamma}{2} \mathrm{e}^{-\frac{\gamma(t-\tau)}{2}} \frac{\sin \left((t-\tau) \sqrt{-\gamma^{2} / 4-\lambda_{k}-h}\right)}{\sqrt{-\gamma^{2} / 4-\lambda_{k}-h}}\right. \\
\left.+\mathrm{e}^{-\frac{\gamma(t-\tau)}{2}} \cos \left((t-\tau) \sqrt{-\gamma^{2} / 4-\lambda_{k}-h}\right)\right] \int_{0}^{1}(-p(x, \tau)) \omega_{k}(x) \mathrm{d} x \mathrm{~d} \tau,
\end{gathered}
$$

where $p(x, t)=F(x, t, y(x, t))$.

The series $(2.3 \mathrm{a}-\mathrm{i})$ converges in $C\left([0, T] ; H_{0}^{1}(0,1)\right) \bigcap C^{1}\left([0, T] ; L^{2}(0,1)\right)$ for every $T>0$.

\section{Proof of Theorem 1.2}

Step 1 (the set of target states). Note that, to prove Theorem 1.2, it is sufficient to do it for any set of non-negative target state $y_{d}$ which is dense in the non-negative part of the space $H_{0}^{1}(0,1)$. To this end, we will further consider only:

(A) non-negative continuously differentiable functions $y_{d}=y_{d}(x), x \in[0,1]$ that

(B) vanish at $x=0, x=1$, i.e., $y_{d}(0)=y_{d}(1)=0$, and

(C) whose second derivatives are piecewise continuous with finitely many discontinuities of the first kind (hence, $\left.y_{d} \in H^{2}(0,1) \bigcap H_{0}^{1}(0,1) \subset C^{1}[0,1]\right)$ and are such that

(D)

$$
y_{d}(x)>0 \text { in }(0,1) \text { and } \frac{y_{d x x}}{y_{d}} \in L^{\infty}(0,1)
$$

To ensure the last condition in (3.1), it is sufficient to select $y_{d}$ such that, in addition to the prior conditions, $y_{d}$ is linear near the endpoints $x=0,1$. This would guarantee that $y_{d x x}=0$ near $x=0,1$, while elsewhere, due to the first condition in (3.1), the denominator in (3.1) is strictly separated from 0 . (In [12] we showed how it can done in detail.)

Further control strategy. We intend to apply the following strategy to prove Theorem 1.2:

- First of all, given the target state $y_{d}$ (selected arbitrarily within the set outlined in Step 1), in Step 2 we intend to choose $v_{*}$ as in (1.2a) such that the first eigenfunction $\omega_{1}$, associated with the spectral problem (1.6a-b), will be co-linear with $y_{d}$ and the first eigenvalue $\lambda_{1}$ will be negative. 
- Then, in Step 3, we intend to apply control $v_{*}+h_{1}$ of the form (1.2a-b) with any $h_{1}<0$ and to show that for some $t_{1}=t_{1}\left(h_{1}\right)$ we can have

$$
u_{1}\left(t_{1}\right) \in\left(0,\left\|y_{d}\right\|_{L^{2}(0,1)}\right)
$$

where $u_{1}(t) \omega_{1}$ stands for the first term in the solution expansion of the form (2.3a) for system (1.8). The key observation here, allowing us to achieve this goal, is the fact that the solution formulas $(2.3 \mathrm{f}-\mathrm{i})$ with $F=0$ describe the ellipsoidal motion of the point $\left(u_{1}(t), \dot{u}_{1}(t)\right)$ in the associated two dimensional space when the aforementioned constant controls are used.

- After that, given the selection of $v_{*}, h_{1}$, and $t_{1}$, we will simply evaluate the difference between $\left(y_{d}, 0\right)$ and $\left(u(t), u_{t}(t)\right)$ in the space $L^{2}(0,1) \times H^{-1}(0,1)$ for $t>t_{1}$. Our goal here is,

(a) making use of a specific choice of the value for $h_{2}$ as given in (1.2a-b)-(1.3);

(b) to apply the effect of carefully designed damping gain $\gamma$ to ensure that;

(c) the above difference can be made as small as we wish at some carefully selected moments of time. The choice of the above parameters is rather technical and is tailored specifically to do it so that the proof of Theorem 1.2 can later be extended to prove Theorem 1.1. Namely, we need to make sure that the corresponding terms in the expansion (2.3a-i) containing $F$ can be suppressed (see, e.g., the key estimates (4.14) and (4.15)).

Step 2 (selection of $\left.v_{*}(x)\right)$. Consider any initial state $\left(y_{0}, y_{1}\right) \in H_{0}^{1}(0,1) \times L^{2}(0,1), \quad\left(y_{0}, y_{1}\right) \neq(0,0)$ and any non-negative target equilibrium state $\left(y_{d}, 0\right), y_{d}(x) \geq 0, y_{d} \in H_{0}^{1}(0,1)$, satisfying the aforementioned conditions (A)-(D).

Select

$$
v_{*}(x)=-\frac{y_{d x x}(x)}{y_{d}(x)}-2\left\|\frac{y_{d x x}}{y_{d}}\right\|_{L^{\infty}(0,1)}, \quad x \in(0,1) .
$$

(Note that the inequality in (1.2a) is satisfied for our choice.)

Since $y_{d}(x)>0$ in $(0,1)$ (see $\left.(3.1)\right)$, condition (3.2) implies that

$$
\frac{y_{d}}{\left\|y_{d}\right\|_{L^{2}(0,1)}}=\omega_{1}
$$

(see also, e.g., [9] for more details) and that

$$
\lambda_{1}=\int_{0}^{1}\left(-\omega_{1 x}^{2}+v_{*} \omega_{1}^{2}\right) \mathrm{d} x=-2\left\|\frac{y_{d x x}}{y_{d}}\right\|_{L^{\infty}(0,1)},<0 .
$$

Remark 3.1. Without loss of generality, we further assume that

$$
\left(\int_{0}^{1} y_{d} y_{0} \mathrm{~d} x\right)^{2}+\left(\int_{0}^{1} y_{d} y_{1} \mathrm{~d} x\right)^{2} \neq 0
$$

(Indeed, otherwise, we just replace $y_{d}$ with an alternative "arbitrarily close" to it target function for which this inequality holds.)

Step 3. (selection of $t_{1}$ for any $h(t)=h_{1}<0$ in $(1.2 \mathrm{~b})$ ). Given the initial datum $\left(y_{0}, y_{1}\right)$, our goal is to select a control pair $\left(h_{1}<0, \gamma=0\right)$ in such way that at some time $t=t_{1}$ the solution expansion for system (1.8) will 
look like (see $(2.3 \mathrm{a}))$ :

$$
\begin{gathered}
u\left(x, t_{1}\right)=u_{1}\left(t_{1}\right) \omega_{1}(x)+\sum_{k=2}^{\infty} u_{k}\left(t_{1}\right) \omega_{k}(x), \text { where } u_{1}\left(t_{1}\right) \in\left(0,\left\|y_{d}\right\|_{L^{2}(0,1)}\right), \\
u_{t}\left(x, t_{1}\right)=\sum_{k=1}^{\infty} \dot{u}_{k}\left(t_{1}\right) \omega_{k}(x) .
\end{gathered}
$$

(Recall that $\left\|y_{d}\right\|_{L^{2}(0,1)} \omega_{1}(x)=y_{d}$.)

Consider any $h_{1}<0$. Then the eigenvalues of the corresponding spectral problem $\omega_{x x}+v \omega=\lambda \omega$, associated with $v(x)=v_{*}(x)+h_{1}$, are as follows:

$$
0>\lambda_{1}+h_{1}>\lambda_{2}+h_{1}>\ldots
$$

while the respective eigenfunctions $\omega_{k}$ 's will remain the same as in the case when $v=v_{*}$ (see (1.6a-b), $(3.3),(3.4))$.

Then (2.3f-i), applied to the solution of (1.8) with $v(x)=v_{*}(x)+h_{1}$ and $\gamma=0$, yield that the point $\left(u_{1}(t), \dot{u}_{1}(t)\right)$ moves with period $2 \pi /\left(-h_{1}-\lambda_{1}\right)^{1 / 2}$ in the associated two dimensional space around the origin along the ellipse

$$
\left|\lambda_{1}+h_{1}\right| u_{1}^{2}(t)+\dot{u}_{1}^{2}(t)=\left|\lambda_{1}+h_{1}\right| u_{1}^{2}(0)+\dot{u}_{1}^{2}(0) .
$$

Hence, we can find a $t_{1}$ such that $u_{1}\left(t_{1}\right) \in\left(0,\left\|y_{d}\right\|_{L^{2}(0,1)}\right)$ as in (3.5a), within one rotation around the origin, that is,

$$
t_{1} \in\left[0, \frac{2 \pi}{\sqrt{-h_{1}-\lambda_{1}}}\right] .
$$

Step 4 (steering for $t>t_{1}$ ). In this step we intend to steer the system (1.8) from the state (3.5a-b), (3.7) "arbitrarily close" in $L^{2}(0,1) \times H^{-1}(0,1)$ to our desirable target state

$$
\left(y_{d}(x), 0\right)
$$

by making use of the following pair of controls of the form $(1.2 \mathrm{a}-\mathrm{b}),(1.3)$ :

$$
h_{2}=-\lambda_{1}+r, \quad \gamma>0, \quad \text { where } r \in\left(0,0.5 \min \left\{1, \lambda_{1}-\lambda_{2}\right\}\right)
$$

and $\gamma$ is "large enough." In other words, we intend to apply damping to all the terms in the expansion (3.5a-b), except for the first term in $(3.5 \mathrm{a})$, which we intend to steer to our target $y_{d}$. Our argument is split below into several steps.

\subsubsection{Selection of $\gamma>0$}

We will now select a special sequence $\left\{\gamma_{i}\right\}_{i=1}^{\infty}, \gamma_{i}>0$ for the values of damping $\gamma$.

Recall that

$$
\lim _{k \rightarrow \infty} \frac{-\lambda_{k}}{k^{2}}=\text { a positive constant. }
$$

This means, in particular, that there exists a sequence $\left\{k_{i}\right\}_{i=1}^{\infty}$ such that

$$
\lambda_{k_{i}}-\lambda_{k_{i}+1} \rightarrow \infty \quad \text { as } i \rightarrow \infty
$$

This allows us to select $\gamma_{i}^{2} \in\left(-4\left(\lambda_{k_{i}}-\lambda_{1}\right),-4\left(\lambda_{k_{i}+1}-\lambda_{1}\right)\right)$ such that

$$
\left|\gamma_{i}^{2}+4\left(\lambda_{k_{i}}-\lambda_{1}\right)\right|,\left|\gamma_{i}^{2}+4\left(\lambda_{k_{i}+1}-\lambda_{1}\right)\right| \rightarrow \infty
$$


as $\gamma_{i} \rightarrow \infty$ by choosing $\gamma_{i}^{2}$ 's as the "midpoints" of the aforementioned intervals:

$$
\begin{gathered}
\gamma_{i}=\sqrt{-4\left(\lambda_{k_{i}}-\lambda_{1}\right)+\frac{1}{2}\left(-4\left(\lambda_{k_{i}+1}-\lambda_{1}\right)-\left(-4\left(\lambda_{k_{i}}-\lambda_{1}\right)\right)\right)} \\
=\sqrt{4 \lambda_{1}-2\left(\lambda_{k_{i}+1}+\lambda_{k_{i}}\right)}, \quad i=1, \ldots
\end{gathered}
$$

Denote

$$
b(\gamma)=\inf _{k=1, \ldots ; r \in\left(0,0.5 \min \left\{1, \lambda_{1}-\lambda_{2}\right\}\right)}\left|\gamma^{2}+4\left(\lambda_{k}-\lambda_{1}+r\right)\right| .
$$

Conditions (3.10a-b) ensure that

$$
b\left(\gamma_{i}\right) \rightarrow \infty \quad \text { as } i \rightarrow \infty
$$

Below we deal only with $\gamma$ 's from the sequence in (3.11), denoting them just by $\gamma$.

\subsubsection{Evaluation of $\left\|u(\cdot, t)-y_{d}\right\|_{L^{2}(0,1)}^{2}$}

We often use below the following formulas:

$$
\begin{gathered}
-\frac{\gamma}{2}+\sqrt{\gamma^{2} / 4+\left(\lambda_{k}-\lambda_{1}+r\right)}=\frac{\lambda_{k}-\lambda_{1}+r}{\gamma / 2+\sqrt{\gamma^{2} / 4+\left(\lambda_{k}-\lambda_{1}+r\right)}}, \\
\mathrm{e}^{\left(-\frac{\gamma}{2}+\sqrt{\gamma^{2} / 4+\left(\lambda_{k}-\lambda_{1}+r\right)}\right)\left(t-t_{1}\right)} \leq \mathrm{e}^{\left(\lambda_{k}-\lambda_{1}+r\right) \frac{t-t_{1}}{\gamma}},
\end{gathered}
$$

for all $t>t_{1}$ whenever $\gamma^{2} / 4+\left(\lambda_{k}-\lambda_{1}+r\right)>0$ and $\lambda_{k}-\lambda_{1}+r<0$.

Denote $Q_{2}=(0,1) \times\left(t_{1}, t\right)$ and let $N(\gamma, r)$ be the largest positive integer $k$ such that $\gamma^{2}+4\left(\lambda_{k}-\lambda_{1}+r\right)>0$ (when this set is nonempty).

Then, the solution formulas (2.3d,f) (applied for $t \geq t_{1}$ with the initial datum (3.5a-b) and the aforementioned pair of controls $\left(v=v_{*}+\left(-\lambda_{1}+r\right), \gamma>0\right)$ along with (1.4), (1.5), (3.9) and (3.11) yield as $\gamma \rightarrow \infty$ :

$$
\begin{aligned}
& \left\|u(\cdot, t)-y_{d}\right\|_{L^{2}(0,1)}^{2}=\|u(\cdot, t)-\| y_{d}\left\|_{L^{2}(0,1)} \omega_{1}\right\|_{L^{2}(0,1)}^{2} \\
& \leq M\left(\mathrm{e}^{\frac{r}{0.5+\sqrt{0.25+r / \gamma^{2}}} \frac{t-t_{1}}{\gamma}} \frac{u_{1}\left(t_{1}\right)}{2}\left(\frac{\gamma}{\sqrt{\gamma^{2}+4 r}}+1\right)-\left\|y_{d}\right\|_{L^{2}(0,1)}\right)^{2} \\
& +M \mathrm{e}^{\frac{2 r}{0.5+\sqrt{0.25+r / \gamma^{2}}}} \frac{t-t_{1}}{\gamma} \frac{\left(\int_{0}^{1} u_{t}\left(s, t_{1}\right) \omega_{1}(s) \mathrm{d} s\right)^{2}}{\gamma^{2}+4 r}+M \mathrm{e}^{-\gamma\left(t-t_{1}\right)} \frac{r^{2}\left(\int_{0}^{1} u\left(s, t_{1}\right) \omega_{1}(s) \mathrm{d} s\right)^{2}}{\left(\gamma^{2}+4 r\right)\left(\gamma / 2+\sqrt{\gamma^{2} / 4+r}\right)^{2}} \\
& +M \sum_{k=2}^{N(\gamma, r)} \mathrm{e}^{2\left(\lambda_{k}-\lambda_{1}+r\right) \frac{t-t_{1}}{\gamma}}\left(\frac{\gamma^{2}}{\gamma^{2}+4\left(\lambda_{k}-\lambda_{1}+r\right)}+1\right)\left(\int_{0}^{1} u\left(s, t_{1}\right) \omega_{k}(s) \mathrm{d} s\right)^{2} \\
& +\sum_{k>N(\gamma, r)} \mathrm{e}^{-\gamma\left(t-t_{1}\right)}\left(\frac{\gamma^{2}}{b(\gamma)}+1\right)\left(\int_{0}^{1} u\left(s, t_{1}\right) \omega_{k}(s) \mathrm{d} s\right)^{2}+M \sum_{k=2}^{\infty} \frac{1}{b(\gamma)}\left(\int_{0}^{1} u_{t}\left(s, t_{1}\right) \omega_{k}(s) \mathrm{d} s\right)^{2}
\end{aligned}
$$

where $M>0$ is some constant. 
Here we used (2.3d) and (3.13a-b) to evaluate the first $N(\gamma, r)$ terms in the expansion like in (3.5a) for the solution to (1.8) for $t>t_{1}$ (i.e., when $\gamma^{2}+4\left(\lambda_{k}-\lambda_{1}+r\right)>0$ ), and (2.3f) for the remaining terms (the case (2.3c) is excluded due to (3.9), (3.10b), (3.11), and (3.12b)).

The difference in the appearance of the first three terms in (3.14), evaluating the first term in this expansion, reflects the fact that only for $k=1$ the expression $\lambda_{k}-\lambda_{1}+r$ is positive (due to (3.9)) and equal to $r$, while it is negative for $k \geq 2$.

We want to show that with $\gamma$ as in (3.11) we can select $t>t_{1}$ and $r$ in (3.9) which will make the right-hand side of (3.14) as small as we wish.

Selection of the duration for control action. From now on, given the sequence of values for $\gamma$ as in (3.11), we will deal with the following specially selected sequence of values for the duration of the control time-intervals $\left(t_{1}, t\right)$ (we will explain why later):

$$
t=t_{1}+\frac{1}{C^{*}(1+K B)\left(B+\left(-\lambda_{1}+0.5\right)^{2}+B K\right)} \gamma \ln \gamma
$$

Then for any $r$ as in (3.9) the 3rd, 5th, and the 6th terms in (3.14) converge to zero as $\gamma \rightarrow \infty$ along (3.11), $(3.12 \mathrm{~b})$, and (3.15) uniformly over $r$ as in (3.9). For the 5 th term we also use the fact that

$$
\mathrm{e}^{-\gamma\left(t-t_{1}\right)} \gamma^{2} \rightarrow 0 \text { as } \gamma \rightarrow \infty
$$

and $t-t_{1} \rightarrow \infty$ along (3.11) and (3.15).

Next we select $r=r(\gamma)$ to make the 1st term on the right in (3.14) to be zero, namely, from the condition

$$
\begin{aligned}
& \mathrm{e}^{\frac{r}{0.5+\sqrt{0.25+r / \gamma^{2}}} \frac{t-t_{1}}{\gamma}} \frac{c_{1}\left(t_{1}\right)}{2}\left(\frac{\gamma}{\sqrt{\gamma^{2}+4 r}}+1\right)= \\
& \quad \gamma^{\frac{r}{0.5+\sqrt{0.25+r / \gamma^{2}}} \frac{1}{C^{*}(1+K B)\left(B+\left(-\lambda_{1}+0.5\right)^{2}+B K\right)}} \frac{c_{1}\left(t_{1}\right)}{2}\left(\frac{1}{\sqrt{1+4 r / \gamma^{2}}}+1\right)=\left\|y_{d}\right\|_{L^{2}(0,1)} .
\end{aligned}
$$

Such $r(\gamma)$ exists for any $\gamma>1$ (recall here (3.11)), because the left-hand side of (3.16a) for $r=0$ is equal to $c_{1}\left(t_{1}\right)$ lying in the interval $\left(0,\left\|y_{d}\right\|_{L^{2}(0,1)}\right)$ (as in (3.5a)) and it tends to $\infty$ as $r \rightarrow \infty$. Moreover, for any $r>0$ the left-hand side of (3.16a) tends to $\infty$ as $\gamma \rightarrow \infty$. Hence, this $r=r(\gamma)$ can be selected to satisfy (3.9), more precisely,

$$
r=r(\gamma) \rightarrow 0 \text { as } \gamma \rightarrow \infty
$$

along (3.11) and $t$ calculated as in (3.15).

We intend now to show that

$$
\begin{gathered}
\sup _{k=2, \ldots, N(\gamma, r)} \mathrm{e}^{2\left(\lambda_{k}-\lambda_{1}+r\right) \frac{t-t_{1}}{\gamma}} \frac{\gamma^{2}}{\gamma^{2}+4\left(\lambda_{k}-\lambda_{1}+r\right)} \rightarrow 0, \\
\frac{1}{\gamma^{2}} \mathrm{e}^{\frac{2 r}{0.5+\sqrt{0.25+r / \gamma^{2}}} \frac{t-t_{1}}{\gamma}} \rightarrow 0
\end{gathered}
$$

under the conditions (3.9), (3.11), (3.15), and (3.16a-b), which will allow us to make the remaining 2nd and 4th terms in (3.14) as small as we wish. 


\subsubsection{Derivation of (3.18)}

Note that for any $\beta \in(0,1)$ we have:

$$
\mathrm{e}^{\frac{2 r}{0.5+\sqrt{0.25+r / \gamma^{2}}} \frac{t-t_{1}}{\gamma}}=\gamma^{\frac{2 r}{0.5+\sqrt{0.25+r / \gamma^{2}}} \frac{1}{0.5+C^{*}(1+K)\left(B+\left(-\lambda_{1}+0.5\right)^{2}+K B\right)}} \leq \gamma^{\beta} \text { as } \gamma \rightarrow \infty
$$

for large $\gamma$ 's in (3.11), due to (3.15),(3.16b), which yields (3.18).

\subsubsection{Derivation of (3.17)}

To show that the right-hand side of (3.17) tend to zero along the conditions (3.9), (3.11), (3.15), and (3.16a-b), note first that for $k=2, \ldots, N(\gamma, r)$ :

$$
\gamma^{2}+4\left(\lambda_{k}-\lambda_{1}+r\right)>0
$$

and

$\sup _{k=2, \ldots, N(\gamma, r)} \mathrm{e}^{2\left(\lambda_{k}-\lambda_{1}+r\right) \frac{t-t_{1}}{\gamma}} \frac{\gamma^{2}}{\gamma^{2}+4\left(\lambda_{k}-\lambda_{1}+r\right)}=\sup _{k=2, \ldots, N(\gamma, r)} \gamma^{\frac{2\left(\lambda_{k}-\lambda_{1}+r\right)}{C^{*}(1+K B)\left(B+\left(-\lambda_{1}+0.5\right)^{2}+B K\right)}} \frac{\gamma^{2}}{\gamma^{2}+4\left(\lambda_{k}-\lambda_{1}+r\right)}$

Furthermore, for all the aforementioned $k$ 's such that

$$
\frac{2\left(\lambda_{k}-\lambda_{1}+r\right)}{C^{*}(1+K B)\left(B+\left(-\lambda_{1}+0.5\right)^{2}+B K\right)}+2 \leq 0
$$

we have:

$$
\gamma^{\frac{2\left(\lambda_{k}-\lambda_{1}+r\right)}{C^{*}(1+K B)\left(B+\left(-\lambda_{1}+0.5\right)^{2}+B K\right)}} \frac{\gamma^{2}}{\gamma^{2}+4\left(\lambda_{k}-\lambda_{1}+r\right)} \leq \frac{1}{b(\gamma)}
$$

For the remaining no more than finitely many $k$ 's, say, $k=2, \ldots, k_{*} \leq N(\gamma, r)$ such that

$$
\frac{2\left(\lambda_{k}-\lambda_{1}+r\right)}{C^{*}(1+K B)\left(B+\left(-\lambda_{1}+0.5\right)^{2}+B K\right)}+2>0
$$

(note that $k_{*}$, if such exists, is bounded above by a number which does not depend on $r$ and $\gamma$ ), we have

$$
\gamma^{\frac{2\left(\lambda_{k}-\lambda_{1}+r\right)}{C^{*}(1+K B)\left(B+\left(-\lambda_{1}+0.5\right)^{2}+B K\right)}} \frac{\gamma^{2}}{\gamma^{2}+4\left(\lambda_{k}-\lambda_{1}+r\right)} \leq \frac{\gamma^{l(r)}}{\gamma^{2}+4\left(\lambda_{k_{*}}-\lambda_{1}+r\right)} \rightarrow 0 \text { as } \gamma \rightarrow \infty \quad \forall k=2, \ldots, k_{*},
$$

where

$$
\begin{aligned}
l(r) & =\max _{k=2, \ldots, k_{*}}\left(\frac{2\left(\lambda_{k}-\lambda_{1}+r\right)}{C^{*}(1+K B)\left(B+\left(-\lambda_{1}+0.5\right)^{2}+B K\right)}+2\right) \\
& <\left(\frac{2\left(\lambda_{2}-\lambda_{1}+0.5 \min \left\{1, \lambda_{1}-\lambda_{2}\right\}\right)}{C^{*}(1+K B)\left(B+\left(-\lambda_{1}+0.5\right)^{2}+B K\right)}+2\right)<2,
\end{aligned}
$$

because $\lambda_{2}-\lambda_{1}+0.5 \min \left\{1, \lambda_{1}-\lambda_{2}\right\}<0$. Combining (3.20)-(3.21) yields (3.17). 
This completes the proof that the right-hand side of (3.14) tends to zero along the relations (3.11), (3.15), (3.9), and (3.16a-b).

\subsubsection{Evaluation of $\left.\| u_{t}(\cdot, t)\right) \|_{H^{-1}(0,1)}$}

Analogously, using the formulas $(2.3 \mathrm{e}, \mathrm{i})$, we obtain for $t \geq t_{1}$ :

$$
\begin{aligned}
& \left\|u_{t}(\cdot, t)\right\|_{H^{-1}(0,1)}^{2} \leq M \frac{1}{-\lambda_{1}} \frac{r^{2}}{\gamma^{2}+4 r} \mathrm{e}^{\frac{2 r}{0.5+\sqrt{0.25+r / \gamma^{2}}} \frac{t-t_{1}}{\gamma}}\left(\int_{0}^{1} u\left(s, t_{1}\right) \omega_{1}(s) \mathrm{d} s\right)^{2} \\
& +M \sum_{k=2}^{N(\gamma, r)} \frac{1}{b(\gamma)} \frac{\left|\lambda_{k}-\lambda_{1}+r\right|^{2}}{-\lambda_{k}}\left(\int_{0}^{1} u\left(s, t_{1}\right) \omega_{k}(s) \mathrm{d} s\right)^{2} \\
& +M \frac{1}{-\lambda_{1}} \frac{1}{\gamma^{2}+4 r} \mathrm{e}^{\frac{2 r}{0.5+\sqrt{0.25+r / \gamma^{2}}} \frac{t-t_{1}}{\gamma}}\left(\frac{r}{0.5 \gamma+\sqrt{0.25 \gamma+r}}\right)^{2}\left(\int_{0}^{1} u_{t}\left(s, t_{1}\right) \omega_{1}(s) \mathrm{d} s\right)^{2} \\
& +M \sum_{k=2}^{N(\gamma, r)} \frac{1}{-\lambda_{k}} \frac{1}{b(\gamma)}\left(\frac{\left|\lambda_{k}-\lambda_{1}+r\right|}{0.5 \gamma+\sqrt{0.25 \gamma+\lambda_{k}-\lambda_{1}+r}}\right)^{2}\left(\int_{0}^{1} u_{t}\left(s, t_{1}\right) \omega_{k}(s) \mathrm{d} s\right)^{2} \\
& +M \frac{1}{-\lambda_{1}}\left(\mathrm{e}^{\left(-\frac{\gamma}{2}-\sqrt{\frac{\gamma^{2}}{4}+r}\right)\left(t-t_{1}\right)}\left(\frac{\gamma}{2}+\sqrt{\frac{\gamma^{2}}{4}+r}\right)\right)^{2} \frac{1}{\gamma^{2}+4 r}\left(\int_{0}^{1} u_{t}\left(s, t_{1}\right) \omega_{1}(s) \mathrm{d} s\right)^{2} \\
& +M \sum_{k=2}^{N(\gamma, r)} \frac{1}{-\lambda_{k}} \frac{1}{b(\gamma)}\left(\mathrm{e}^{\left(-\frac{\gamma}{2}-\sqrt{\frac{\gamma^{2}}{4}+\lambda_{k}-\lambda_{1}+r}\right)\left(t-t_{1}\right)}\left(\frac{\gamma}{2}+\sqrt{\frac{\gamma^{2}}{4}+\lambda_{k}-\lambda_{1}+r}\right)\right)^{2}\left(\int_{0}^{1} u_{t}\left(s, t_{1}\right) \omega_{k}(s) \mathrm{d} s\right)^{2} \\
& +M \sum_{k=N(\gamma, r)+1}^{\infty} \frac{1}{-\lambda_{k}} \mathrm{e}^{-\gamma\left(t-t_{1}\right)}\left(\frac{\gamma^{2}}{4}+\left|\lambda_{k}-\lambda_{1}+r\right|+\frac{\gamma^{4}}{b(\gamma)}\right)\left(\int_{0}^{1} u\left(s, t_{1}\right) \omega_{k}(s) \mathrm{d} s\right)^{2} \\
& +M \sum_{k=N(\gamma, r)+1}^{\infty} \frac{1}{-\lambda_{k}} \mathrm{e}^{-\gamma\left(t-t_{1}\right)}\left(1+\frac{\gamma^{2}}{b(\gamma)}\right)\left(\int_{0}^{1} u_{t}\left(s, t_{2}\right) \omega_{1}(s) \mathrm{d} s\right)^{2}
\end{aligned}
$$

where $M>0$ is some positive constant.

An argument similar to that we applied to evaluate (3.14) implies that the right-hand side in (3.22) tends to zero as $\gamma \rightarrow \infty$ under the conditions (3.11), (3.15), (3.9), and (3.16a-b).

In particular, (3.18) can be used to evaluate the 1 st and the $3 r d$ terms.

To evaluate the $2 n d$ and the 4 th terms we can use $(3.12 \mathrm{~b})$ along with the following observations.

Recalling that $u\left(\cdot, t_{1}\right) \in H_{0}^{1}(0,1)$, we have:

$$
\sum_{k=1}^{\infty}\left|\lambda_{k}\right|\left(\int_{0}^{1} u\left(s, t_{1}\right) \omega_{k}(s) \mathrm{d} s\right)^{2}<\infty
$$


and, hence, we can evaluate the 2nd term as

$$
\begin{aligned}
M \sum_{k=2}^{N(\gamma, r)} \frac{1}{b(\gamma)} \frac{\left|\lambda_{k}-\lambda_{1}+r\right|^{2}}{-\lambda_{k}}\left(\int_{0}^{1} u\left(s, t_{1}\right) \omega_{k}(s) \mathrm{d} s\right)^{2} \leq \\
M \sum_{k=2}^{N(\gamma, r)} \frac{1}{b(\gamma)} \frac{\left|\lambda_{k}-\lambda_{1}+r\right|^{2}}{-\lambda_{k}^{2}}\left(-\lambda_{k}\left(\int_{0}^{1} u\left(s, t_{1}\right) \omega_{k}(s) \mathrm{d} s\right)^{2}\right) .
\end{aligned}
$$

To evaluate the $\underline{4 \text {-th term }}$, note that, uniformly over $k=2, \ldots, N(\gamma, r)$,

$$
\begin{aligned}
\frac{1}{-\lambda_{k}} \frac{1}{b(\gamma)}\left(\frac{\left|\lambda_{k}-\lambda_{1}+r\right|}{0.5 \gamma+\sqrt{0.25 \gamma^{2}+\lambda_{k}-\lambda_{1}+r}}\right)^{2} & \leq \frac{1}{b(\gamma)}\left(\frac{\left(-\lambda_{k}\right)^{1 / 2}+\left|-\lambda_{1}+r\right|\left(-\lambda_{k}\right)^{-1 / 2}}{0.5 \gamma}\right)^{2} \\
& \leq \frac{1}{b(\gamma)}\left(\frac{\left(0.25 \gamma^{2}-\lambda_{1}+r\right)^{1 / 2}+\left|-\lambda_{1}+r\right|\left(-\lambda_{k}\right)^{-1 / 2}}{0.5 \gamma}\right)^{2} \rightarrow 0
\end{aligned}
$$

as $\gamma \rightarrow \infty$, because

$$
0<-\lambda_{k}<0.25 \gamma^{2}-\lambda_{1}+r
$$

for our $k=2, \ldots, N(\gamma, r)$.

To evaluate the remaining $5 t h, 6 t h, 7 t h$, and 8 th terms, we can use the fact that

$$
\mathrm{e}^{-\gamma\left(t-t_{1}\right)} \gamma^{\kappa} \rightarrow 0 \quad \text { as } \gamma \rightarrow \infty \text { and } t-t_{1} \rightarrow \infty
$$

along (3.11) and (3.15) for any $\kappa \geq 0$ (and also the fact that $\lambda_{k}-\lambda_{1}+r<0$ for the 6th term).

Therefore, there is a pair of controls $(v, \gamma)$ and time $T>0$ described in Theorem 1.2 such that (1.9) holds. This ends the proof of Theorem 1.2.

\section{Proof of Theorem 1.1}

Our plan is simply to show that we can further modify the control pair $(v, \gamma)$ within the restrictions imposed on it in the proof of Theorem 1.2 in such a way that they would generate a trajectory of the original system (S) that follows "closely" to that of $(1.8)$, and, thus, system $(\mathrm{S})$ will arrive to the same desirable target $\left(y_{d}, 0\right)$ as (1.8) as well.

The key observation here is that the evaluation of the difference between $\left(y, y_{t}\right)$ and $\left(u, u_{t}\right)$ can be reduced to the evaluation of the terms in $(2.3 \mathrm{a}-\mathrm{i})$ containing $F$.

\subsection{Some preliminary estimates}

Lemma 4.1. Let $h \geq 0$ and $\gamma>0$ be given real numbers. Then the following estimate holds for system (S), (1.4a-b), (1.5) and (1.2a) with constant $h(t)=h$ for all $t>0$ :

$$
\int_{0}^{1} y^{2}(x, t) \mathrm{d} x \leq 2 C^{*} E(y(\cdot, t)) \leq 2 C^{*}\left(E(y(\cdot, 0))+\frac{\rho}{2} L B\right) \mathrm{e}^{\rho C^{*}(1+K B) t} \forall \rho \geq \frac{1}{2 \gamma}\left(B+h^{2}+K B\right),
$$

where $C^{*}$ is a positive constant from Poincare's inequality (see (4.3) below) and $K, L$, and $B$ are from (1.5). 
Proof. Note first that, due to the inequality $a b \leq \rho a^{2} / 2+b^{2} /(2 \rho)(\forall \rho>0)$,

$$
h y y_{t} \leq \frac{\rho}{2} y^{2}+\frac{h^{2}}{2 \rho} y_{t}^{2}
$$

and, due to (1.5),

$$
\iint_{Q_{t}}(1+|y|) g\left|y_{t}\right| \mathrm{d} x \mathrm{~d} \tau \leq \frac{\rho}{2} L+\iint_{Q_{t}}\left(\frac{1}{2 \rho} y_{t}^{2}+K \frac{\rho}{2} y^{2}+\frac{K}{2 \rho} y_{t}^{2}\right) \mathrm{d} x \mathrm{~d} \tau
$$

where $Q_{t}=(0,1) \times(0, t)$.

Multiply the equation in $(\mathrm{S})$ by $y_{t}$ and integrate the resulting expression over $Q_{t}$. Then, after standard calculations and making use of the above inequalities, we obtain the following:

$$
\begin{aligned}
E(y(\cdot, t)) \leq & E(y(\cdot, 0))+h \iint_{Q_{t}} y y_{t} \mathrm{~d} x \mathrm{~d} \tau-\gamma \iint_{Q_{t}} y_{t}^{2} \mathrm{~d} x \mathrm{~d} \tau+\iint_{Q_{t}} B(1+|y|) g\left|y_{t}\right| \mathrm{d} x \mathrm{~d} \tau \\
& \leq E(y(\cdot, 0))+\frac{\rho}{2} L B+\frac{\rho}{2}(1+K B) \iint_{Q_{t}} y^{2} \mathrm{~d} x \mathrm{~d} \tau+\left(\frac{1}{2 \rho}\left(B+h^{2}+K B\right)-\gamma\right) \iint y_{t}^{2} \mathrm{~d} x \mathrm{~d} \tau \\
& \leq\left(E(y(\cdot, 0))+\frac{\rho}{2} L B\right)+C^{*} \rho(1+K B) \int_{0}^{t} E\left(y(\cdot, \tau) \mathrm{d} \tau \quad \forall \gamma \geq \frac{1}{2 \rho}\left(B+h^{2}+K B\right),\right.
\end{aligned}
$$

where $C^{*}$ is the constant in Poincare's inequality:

$$
\int_{0}^{1} \phi^{2}(x) \mathrm{d} x \leq C^{*} \int_{0}^{1} \phi_{x}^{2}(x) \mathrm{d} x \quad \forall \phi \in H_{0}^{1}(0,1)
$$

Applying Gronwall-Bellman inequality to (4.2) yields the 2nd inequality in (4.1). To complete the proof of Lemma 4.1, note that the 1st inequality in (4.1) is just an immediate consequence of (4.3).

Lemma 4.2. Let $t>0$ be given. Then the following estimate holds for system (S), (1.4a-b), (1.5) and (1.2a) with any constant $h(t)=h<0$ and $\gamma=0$ :

$$
\begin{aligned}
& \int_{0}^{1} y^{2}(x, \tau) \mathrm{d} x \leq \frac{1}{-h} E(y(\cdot, 0))+\frac{L B}{-2 h}+\int_{0}^{1} y_{0}^{2}(x) \mathrm{d} x \\
& +B\left(1+K+C^{*} K\right) t\left(\frac{1}{-h} E(y(\cdot, 0))+\frac{L B}{-2 h}+\int_{0}^{1} y_{0}^{2}(x) \mathrm{d} x\right) \mathrm{e}^{B\left(1+K+C^{*} K\right) t} \forall \tau \in[0, t]
\end{aligned}
$$

where $C^{*}$ is from (4.3) and $L, K$, and $B$ are from (1.5).

Proof. Indeed, similar to (4.2) (but with $\rho=1$ ) for any $\tau \in[0, t]$ :

$$
E(y(\cdot, \tau))-h \int_{0}^{1} y^{2}(x, \tau) \mathrm{d} x \leq\left(E(y(\cdot, 0))+\frac{L B}{2}\right)-h \int_{0}^{1} y_{0}^{2}(x) \mathrm{d} x+B\left(1+K+C^{*} K\right) \int_{0}^{\tau} E(y(\cdot, s) \mathrm{d} s .
$$


Applying Gronwall-Bellman inequality to (4.5) yields:

$$
E(y(\cdot, \tau)) \leq\left(E(y(\cdot, 0))+\frac{L B}{2}-h \int_{0}^{1} y_{0}^{2}(x) \mathrm{d} x\right) \mathrm{e}^{B\left(1+K+C^{*} K\right) t} \forall \tau \in[0, t] .
$$

Dividing (4.5) by $-h>0$ and making use of (4.6) yields (4.4). This ends the proof of Lemma 4.2.

\subsection{Proof of Theorem $\mathbf{1 . 1}$}

Step 1 (steering on $\left(0, t_{1}\right)$ and selection of $\left.h_{1}\right)$. In Step 3 in the proof of Theorem 1.2 we showed that, given $y_{d}$ and the initial datum $\left(y_{0}, y_{1}\right)$, for any control pair $\left(h_{1}, \gamma=0\right)$, where $h_{1}$ is an arbitrary negative number, we can find the moment $t=t_{1}$ as in (3.7) such that (3.5a-b) holds.

Noting now that the solution expansion (2.3a) for (S) at time $t=t_{1}$ looks like

$$
y\left(x, t_{1}\right)=c_{1}\left(t_{1}\right) \omega_{1}(x)+\sum_{k=2}^{\infty} c_{k}\left(t_{1}\right) \omega_{k}(x), y_{t}\left(x, t_{1}\right)=\sum_{k=1}^{\infty} \dot{c}_{k}\left(t_{1}\right) \omega_{k}(x),
$$

we will now try to select the value for $h_{1}$ such that the solution of (S) would follow "closely" the corresponding solution to (1.8) when the same controls $v=v_{*}+h_{1}, \gamma=0$ are applied.

To this end, we will evaluate the difference between $\left(u_{1}(t), \dot{u}_{1}(t)\right)$ and $\left(c_{1}(t), \dot{c}_{1}(t)\right)$ on $\left[0,2 t_{1}^{*}\right]$, where

$$
t_{1}^{*}=\frac{2 \pi}{\sqrt{-h_{1}-\lambda_{1}}}
$$

is the period of one rotation of the point $\left(u_{1}(t), \dot{u}_{1}(t)\right)$ about the origin as in (3.6).

First of all, note that the function

$$
z(x, t)=y(x, t)-u(x, t)
$$

satisfies the following mixed problem:

$$
\begin{gathered}
z_{t t}=z_{x x}+\left(v_{*}(x)+h_{1}\right) z-\gamma(t) z_{t}-q(t) f(x, t, y(x, t)), \quad(x, t) \in Q_{2 t_{1}^{*}}=(0,1) \times\left(0,2 t_{1}^{*}\right), \\
z(0, t)=z(1, t)=0, \quad z(x, 0)=0, \quad z_{t}(x, 0)=0 .
\end{gathered}
$$

Applying (2.3f-i) now to (4.8), we obtain the following chain of inequalities for every $t \in\left[0,2 t_{1}^{*}\right]$ :

$$
\begin{aligned}
\frac{1}{2}\left(| \lambda _ { 1 } | \left(c_{1}(t)\right.\right. & \left.\left.-u_{1}(t)\right)^{2}+\left(\dot{c}_{1}(t)-\dot{u}_{1}(t)\right)^{2}\right) \\
\leq & \frac{1}{2} \sum_{k=1}^{\infty}\left(-\lambda_{k}\right)\left(\int_{0}^{2 t_{1}^{*}}\left[\frac{\sin \left((t-\tau) \sqrt{-h_{1}-\lambda_{k}}\right)}{\sqrt{-h_{1}-\lambda_{k}}} \int_{0}^{1}(-q(\tau) f(x, t, y(x, \tau))) \omega_{k}(x) \mathrm{d} x\right] \mathrm{d} \tau\right)^{2} \\
& +\frac{1}{2} \sum_{k=1}^{\infty}\left(\int_{0}^{2 t_{1}^{*}}\left[\cos \left((t-\tau) \sqrt{-h_{1}-\lambda_{k}}\right) \int_{0}^{1}(-q(\tau) f(x, t, y(x, \tau))) \omega_{k}(x) \mathrm{d} x\right] \mathrm{d} \tau\right)^{2} \\
& \leq 2 t_{1}^{*} B^{2} \int_{Q_{2 t_{1}^{*}}} f^{2} \mathrm{~d} x \mathrm{~d} t .
\end{aligned}
$$


In turn, $(1.4,(1.5),(2.2 \mathrm{~b})$, and (4.4) yield:

$$
\begin{aligned}
\iint_{Q_{2 t_{1}^{*}}} f^{2} \mathrm{~d} x \mathrm{~d} t \leq 4 K^{2} t_{1}^{*}\left\{1+\frac{1}{-h_{1}} E(y(\cdot, 0))+\frac{L B}{-2 h_{1}}+\int_{0}^{1} y_{0}^{2}(x) \mathrm{d} x\right. \\
\left.+B\left(1+K+C^{*} K\right) 2 t_{1}^{*}\left(\frac{1}{-h_{1}} E(y(\cdot, 0))+\frac{L B}{-2 h_{1}}+\int_{0}^{1} y_{0}^{2}(x) \mathrm{d} x\right) \mathrm{e}^{B\left(1+K+C^{*} K\right) 2 t_{1}^{*}}\right\}
\end{aligned}
$$

Due to (4.10), with $h_{1} \rightarrow-\infty$, the right-hand side of (4.9) tends to zero uniformly over $t \in\left[0,2 t_{1}^{*}\right]$. In other words, the point $\left(c_{1}(t), \dot{c}_{1}(t)\right)$ follows "arbitrarily closely" the two subsequent periodic elliptic motions of the point $\left(u_{1}(t), \dot{u}_{1}(t)\right)$ around the origin in the associated two dimensional space. Hence, there exists a pair $\left(h_{1}<0, t_{1}>0\right)$ such that $(3.5 \mathrm{a}-\mathrm{b})$ holds for the corresponding solution to $(\mathrm{S})$ as well, namely,

$$
c_{1}\left(t_{1}\right) \in\left(0,\left\|y_{d}\right\|_{L^{2}(0,1)}\right)
$$

Step 2 (steering for $t>t_{1}$ ).

\subsubsection{Evaluation of $\left\|y(\cdot, t)-y_{d}\right\|_{L^{2}(0,1)}^{2}$}

Denote by $u\left(x, t, y\left(x, t_{1}\right)\right)$ solution to (1.8) with the initial datum the same as for $(\mathrm{S})$ at $t=t_{1}$, namely, (4.7), (4.11).

Remark 4.1. From now on when we will be referring to the results in section 3 for $t>t_{1}$ we will assume that we deal with the just-introduced initial conditions for $u$ at time $t=t_{1}$.

The solution formulas $(2.3 \mathrm{~d}, \mathrm{f})$, applied for $t \geq t_{1}$ with the initial datum $(4.7),(4.11)$ and the aforementioned pair of controls $v=v_{*}+\left(-\lambda_{1}+r\right), \gamma>0$ as in the proof of Theorem 1.2 (see (3.9)), along with (1.4), (1.5), and (3.11) yield as $\gamma \rightarrow \infty$ :

$$
\begin{array}{r}
\left\|y(\cdot, t)-y_{d}\right\|_{L^{2}(0,1)}^{2}=\|y(\cdot, t)-\| y_{d}\left\|_{L^{2}(0,1)} \omega_{1}\right\|_{L^{2}(0,1)}^{2} \\
\leq\left\|y(\cdot, t)-u\left(x, t, y\left(\cdot, t_{1}\right)\right)\right\|_{L^{2}(0,1)}^{2}+\left\|u\left(x, t, y\left(\cdot, t_{1}\right)\right)-\right\| y_{d}\left\|_{L^{2}(0,1)} \omega_{1}\right\|_{L^{2}(0,1)}^{2} \\
\leq M D \frac{1}{\gamma^{2}} \mathrm{e}^{\frac{2 r}{0.5+\sqrt{0.25+r / \gamma^{2}}} \frac{t-t_{1}}{\gamma}} \iint_{Q_{2}} f^{2}(x, \tau, y(x, \tau)) \mathrm{d} x \mathrm{~d} \tau+M \frac{D}{b(\gamma)} \iint_{Q_{2}} f^{2}(x, \tau, y(x, \tau)) \mathrm{d} x \mathrm{~d} \tau \\
+\left\|u\left(x, t, y\left(\cdot, t_{1}\right)\right)-\right\| y_{d}\left\|_{L^{2}(0,1)} \omega_{1}\right\|_{L^{2}(0,1)}^{2}
\end{array}
$$

where $M>0$ is some constant.

Indeed, to evaluate

$$
\left\|y(\cdot, t)-u\left(x, t, y\left(\cdot, t_{1}\right)\right)\right\|_{L^{2}(0,1)}^{2}
$$

in (4.12) we just need to evaluate the contribution of the terms containing $F$ in $(2.3 \mathrm{~d}, \mathrm{f})$. 
For example, for $k=1,(2.3 \mathrm{~d})$ yields with our $h=-\lambda_{1}+r$ from (3.9):

$$
\begin{aligned}
& \int_{t_{1}}^{t}\left[\mathrm{e}^{\left(-\frac{\gamma}{2}+\sqrt{\gamma^{2} / 4+\lambda_{k}+h}\right)(t-\tau)} \frac{1}{\sqrt{\gamma^{2}+4\left(\lambda_{k}+h\right)}}\right. \\
& \left.\left.-\mathrm{e}^{\left.\left(-\frac{\gamma}{2}-\sqrt{\gamma^{2} / 4+\lambda_{k}+h}\right)(t-\tau)\right)} \frac{1}{\sqrt{\gamma^{2}+4\left(\lambda_{k}+h\right)}}\right] \int_{0}^{1}(-p(x, \tau)) \omega_{k}(x) \mathrm{d} x \mathrm{~d} \tau\right)^{2} \\
& \leq 2\left(\frac{1}{\sqrt{\gamma^{2}+4 r}} \mathrm{e}^{\left(-\frac{\gamma}{2}+\sqrt{\gamma^{2} / 4+r}\right)\left(t-t_{1}\right)} \int_{t_{1}}^{t} q(\tau)\left(\int_{0}^{1} f(x, \tau, y(x, \tau)) \omega_{1}(x) \mathrm{d} x\right) \mathrm{d} \tau\right)^{2} \\
& +2\left(\frac{1}{\sqrt{\gamma^{2}+4 r}} \int_{t_{1}}^{t} q(\tau)\left(\int_{0}^{1} f(x, \tau, y(x, \tau)) \omega_{1}(x) \mathrm{d} x\right) \mathrm{d} \tau\right)^{2} \\
& \leq 2 D\left(\frac{1}{\gamma^{2}} \mathrm{e}^{\frac{2 r}{0.5+\sqrt{0.25+r / \gamma^{2}}} \frac{t-t_{1}}{\gamma}}+\min \left\{\frac{1}{\gamma^{2}}, \frac{1}{b(\gamma)}\right\}\right) \iint_{Q_{2}} f^{2}(x, \tau, y(x, \tau)) \mathrm{d} x \mathrm{~d} \tau,
\end{aligned}
$$

which, in view of (3.13a-b) and (3.12a), provides the 1-st term on the right in (4.12).

Analogously, making use of (2.3d) for $k=2, \ldots, N(\gamma, r)$ when $\gamma^{2}+4\left(\lambda_{k}-\lambda_{1}+r\right)>0$, and of (2.3f), we can obtain the 2 nd term on the right in (4.12).

Then, exactly, as in the proof of Theorem 1.2 beginning from Section 3.4.2, but now with the initial condition as in (4.7), 4.11) at time $t=t_{1}$, we obtain that the last term on the right of (4.12) tends to zero along the relations (3.11), (3.15), (3.9), and (3.16a-b).

To show that the remaining two terms containing $f$ also converge to zero, it is sufficient to show that along the relations (3.11), (3.15), (3.9), and (3.16a-b) the value of $\|f\|_{L^{2}\left(Q_{2}\right)}$ is uniformly bounded, e.g., as follows:

$$
\iint_{Q_{2}} f^{2}(x, \tau, y(x, \tau)) \mathrm{d} x \mathrm{~d} \tau \leq 2 L\left(1+8\left\|y_{d}\right\|_{L^{2}(0,1)}+\frac{4}{-\lambda_{2}+\lambda_{1}-r}\left(E\left(y\left(\cdot, t_{1}\right)+\frac{1}{2}\right)\right),\right.
$$

and that

$$
\frac{1}{\gamma^{2}} \mathrm{e}^{\frac{2 r}{0.5+\sqrt{0.25+r / \gamma^{2}}} \frac{t-t_{1}}{\gamma}} \iint_{Q_{2}} f^{2}(x, \tau, y(x, \tau)) \mathrm{d} x \mathrm{~d} \tau \rightarrow 0
$$

along the same relations.

\subsubsection{Derivation of (4.15)}

We make use of (4.1) from Lemma 2.1 and of (2.2a), (1.4), (1.5):

$$
\begin{aligned}
\iint_{Q_{2}} f^{2}(x, \tau, y(x, \tau)) \mathrm{d} x \mathrm{~d} \tau \leq 2 \iint_{Q_{2}} g^{2}(\tau) \mathrm{d} x \mathrm{~d} \tau & +2 \max _{\tau \in\left[t_{1}, t\right]}\left(\int_{0}^{1} y^{2} \mathrm{~d} x\right) \int_{t_{1}}^{t} g^{2}(\tau) \mathrm{d} \tau \\
& \left.\leq 2 L+4 L C^{*}\left(E\left(y\left(\cdot, t_{1}\right)\right)+\frac{\rho}{2} L B\right)\right) \mathrm{e}^{\rho C^{*}(1+K B)\left(t-t_{1}\right)} \forall t>t_{1}
\end{aligned}
$$


and for $\rho=\frac{1}{2 \gamma}\left(B+\left(-\lambda_{1}+r\right)^{2}+K B\right)$. Hence,

$$
\begin{aligned}
& \iint_{Q_{2}} f^{2}(x, \tau, y(x, \tau)) \mathrm{d} x \mathrm{~d} \tau \leq \\
& \begin{array}{r}
2 L+4 L C^{*}\left(E\left(y\left(\cdot, t_{1}\right)\right)+\frac{1}{4 \gamma} L B\left(B+\left(-\lambda_{1}+r\right)^{2}+K B\right)\right) \mathrm{e}^{C^{*}(1+K B)\left(B+\left(\lambda_{1}+0.5\right)^{2}+K B\right) \frac{t-t_{1}}{2 \gamma}} \\
\quad \leq 2 L+4 L C^{*}\left(E\left(y\left(\cdot, t_{1}\right)\right)+\frac{1}{4 \gamma} L B\left(B+\left(-\lambda_{1}+r\right)^{2}+K B\right)\right) \gamma^{1 / 2}
\end{array}
\end{aligned}
$$

as $\gamma \rightarrow \infty$ along (3.11), due to the formula (recall (3.15))

$$
\mathrm{e}^{\frac{t-t_{1}}{\gamma}}=\gamma^{\frac{1}{C^{*}(1+K B)\left(B+\left(-\lambda_{1}+0.5\right)^{2}+B K\right)}} .
$$

This yields (4.15), by making use of (3.19).

\subsubsection{Derivation of (4.14)}

Note first that (3.16a-b), (3.18) and already established (4.15) mean, as it follows from the first three terms in (3.14) (rewritten for our new initial datum (4.7), (4.11)) and the estimates (4.13) (combined with (4.15)), evaluating $\left|c_{1}(t)-\left\|y_{d}\right\|_{L^{2}(0,1)}\right|$ in (4.12), that the value of $\left|c_{1}(t)\right|$ will be bounded as long as we select $r, t$ and $\gamma \rightarrow \infty$ from (3.9), (3.11), (3.15) and (3.16a-b). Without loss of generality, we can assume that starting with some $\gamma_{*}$,

$$
\left|c_{1}(t)\right| \leq 2\left\|y_{d}\right\|_{L^{2}(0,1)} \text {. }
$$

Multiplying equations $(2.3 \mathrm{~b})$ by $\dot{c}_{k}(t)$ 's, then integrating them over $\left(t_{1}, t\right)$ and summing the results, we obtain:

$$
\begin{aligned}
\frac{1}{2} \sum_{k=1}^{\infty} \dot{c}_{k}^{2}(t)+\frac{1}{2} \sum_{k=2}^{\infty}\left(-\lambda_{k}+\lambda_{1}-r\right) c_{k}^{2}(t) \leq \frac{1}{2} \sum_{k=1}^{\infty} \dot{c}_{k}^{2}\left(t_{1}\right) & +\frac{1}{2} \sum_{k=2}^{\infty}\left(-\lambda_{k}\right) c_{k}^{2}\left(t_{1}\right) \\
& -\gamma \iint y_{t}^{2} \mathrm{~d} x \mathrm{~d} \tau+\iint_{Q_{2}} B g(\tau)(1+|y|)\left|y_{t}\right| \mathrm{d} x \mathrm{~d} \tau .
\end{aligned}
$$

Note that, for any $\nu>0$,

$$
\begin{aligned}
\iint_{Q_{2}} B g(\tau)(1+|y|)\left|y_{t}\right| \mathrm{d} x \mathrm{~d} \tau & \leq \frac{\nu}{2} \iint_{Q_{2}}(B g(\tau)(1+|y|))^{2} \mathrm{~d} x \mathrm{~d} \tau+\frac{1}{2 \nu} \iint_{Q_{2}} y_{t}^{2} \mathrm{~d} x \mathrm{~d} \tau \\
& \leq \frac{\nu}{2} \iint_{Q_{2}} 2 B^{2} g^{2}(\tau) \mathrm{d} x \mathrm{~d} \tau+\frac{\nu}{2} \iint_{Q_{2}} 2 B^{2} g^{2}(\tau) y^{2} \mathrm{~d} x \mathrm{~d} \tau+\frac{1}{2 \nu} \iint_{Q_{2}} y_{t}^{2} \mathrm{~d} x \mathrm{~d} \tau \\
& \leq \nu L B^{2}+\nu L B^{2} \max _{\tau \in\left[t_{1}, t\right]} \int_{0}^{1} y^{2}(x, \tau) \mathrm{d} x+\frac{1}{2 \nu} \iint_{Q_{2}} y_{t}^{2} \mathrm{~d} x \mathrm{~d} \tau
\end{aligned}
$$

Hence, making use of (4.16) and (4.18), and selecting

$$
\nu=\frac{-\lambda_{2}+\lambda_{1}-r}{4 L B^{2}} \quad \text { (we will need this next) }
$$


and $\gamma \geq \frac{1}{2 \nu}$, to get rid of the terms containing $y_{t}$ in (4.17)-(4.18), we obtain from (4.17) that:

$$
\begin{aligned}
c_{k}^{2}(t)+\sum_{k=2}^{\infty} c_{k}^{2}(t) & =\int_{0}^{1} y^{2}(x, t) \mathrm{d} x \leq 4\left\|y_{d}\right\|_{L^{2}(0,1)}^{2}+\frac{2}{-\lambda_{2}+\lambda_{1}-r} \frac{1}{2} \sum_{k=2}^{\infty}\left(-\lambda_{k}+\lambda_{1}-r\right) c_{k}^{2}(t) \\
& \leq 4\left\|y_{d}\right\|_{L^{2}(0,1)}^{2}+\frac{2}{-\lambda_{2}+\lambda_{1}-r} E\left(y\left(\cdot, t_{1}\right)+\frac{2}{-\lambda_{2}+\lambda_{1}-r} \nu L B^{2}\left(1+\max _{\tau \in\left[t_{1}, t\right]} \int_{0}^{1} y^{2}(x, \tau) \mathrm{d} x\right) .\right.
\end{aligned}
$$

Therefore, for $\gamma \geq \frac{1}{2 \nu}$ and our selected $\nu$ we will have

$$
\int_{0}^{1} y^{2}(x, t) \mathrm{d} x \leq 8\left\|y_{d}\right\|_{L^{2}(0,1)}^{2}+\frac{4}{-\lambda_{2}+\lambda_{1}-r}\left(E\left(y\left(\cdot, t_{1}\right)+\frac{1}{2}\right) .\right.
$$

This, similar to (2.2a), yields (4.14).

\subsubsection{Evaluation of $\left.\| y_{t}(\cdot, t)\right) \|_{H^{-1}(0,1)}$}

Making use of the formulas (2.3e, i), similar to the derivation of (4.12), we obtain for $t \geq t_{1}$ :

$$
\begin{aligned}
& \left\|y_{t}(\cdot, t)\right\|_{H^{-1}(0,1)}^{2} \leq\left\|y_{t}(\cdot, t)-u_{t}\left(\cdot, t, y\left(\cdot, t_{1}\right)\right)\right\|_{H^{-1}(0,1)}^{2}+\left\|u_{t}\left(\cdot, t, y\left(\cdot, t_{1}\right)\right)\right\|_{H^{-1}(0,1)}^{2} \\
& \qquad \frac{M}{b(\gamma)} \sup _{\tau \in\left(t_{1}, t\right)}\|f(\cdot, \tau, y(\cdot, \tau))\|_{L^{2}(0,1)}^{2} \sum_{k=2}^{\infty} \frac{B^{2}}{-\lambda_{k}}+\frac{M B^{2}}{\gamma^{2}+4 r} e^{\frac{2 r}{0.5+\sqrt{0.25+r / \gamma^{2}}} \frac{t-t_{1}}{\gamma}} \sup _{\tau \in\left(t_{1}, t\right)}\|f(\cdot, \tau, y(\cdot, \tau))\|_{L^{2}(0,1)}^{2} \\
& \quad+M\left(\frac{1}{b(\gamma)}+\frac{1}{\gamma^{2}}\right) \sup _{\tau \in\left(t_{1}, t\right)}\|f(\cdot, \tau, y(\cdot, \tau))\|_{L^{2}(0,1)}^{2} \sum_{k>N(\gamma, r)}^{\infty} \frac{B^{2}}{-\lambda_{k}}+\left\|u_{t}\left(\cdot, t, y\left(\cdot, t_{1}\right)\right)\right\|_{H^{-1}(0,1)}^{2}
\end{aligned}
$$

where $M>0$ is some positive constant.

In $(4.20)$, to evaluate the terms in $(2.3 \mathrm{e}, \mathrm{i})$ the containing $f$, we also employed the following estimates:

$$
\begin{gathered}
\left|\int_{t_{1}}^{t} a \mathrm{e}^{a(t-\tau)} \mathrm{d} \tau\right|=1-\mathrm{e}^{a\left(t-t_{1}\right)} \leq 1 \quad \forall t \geq t_{1}, a<0, \\
0<\int_{t_{1}}^{t} a \mathrm{e}^{a(t-\tau)} \mathrm{d} \tau=\mathrm{e}^{a\left(t-t_{1}\right)}-1 \forall t \geq t_{1}, a>0, \\
\left|\int_{0}^{1} f(x, t) \omega_{k}(x) \mathrm{d} x\right| \leq\left(\int_{0}^{1} f^{2}(x, t) \mathrm{d} x\right)^{1 / 2}\left(\int_{0}^{1} \omega_{k}^{2}(x) \mathrm{d} x\right)^{1 / 2}=\left(\int_{0}^{1} f^{2}(x, t) \mathrm{d} x\right)^{1 / 2} .
\end{gathered}
$$


For example (see $(2.3 \mathrm{i})$ on $\left.\left(t_{1}, t\right)\right)$ :

$$
\begin{gathered}
\frac{1}{-\lambda_{k}}\left(\int _ { t _ { 1 } } ^ { t } \left[-\frac{\gamma}{2} \mathrm{e}^{-\frac{\gamma(t-\tau)}{2}} \frac{\sin \left((t-\tau) \sqrt{-\gamma^{2} / 4-\lambda_{k}-h}\right)}{\sqrt{-\gamma^{2} / 4-\lambda_{k}-h}}\right.\right. \\
\left.\left.+\mathrm{e}^{-\frac{\gamma(t-\tau)}{2}} \cos \left((t-\tau) \sqrt{-\gamma^{2} / 4-\lambda_{k}-h}\right)\right] \int_{0}^{1}(-p(x, \tau)) \omega_{k}(x) \mathrm{d} x \mathrm{~d} \tau\right)^{2} \\
\leq \frac{2}{-\lambda_{k}} \frac{B^{2}}{-\gamma^{2} / 4-\lambda_{k}+\lambda_{1}-r} \sup _{\tau \in\left(t_{1}, t\right)}\|f(\cdot, \tau, y(\cdot, \tau))\|_{L^{2}(0,1)}^{2}\left(\int_{t_{1}}^{t}\left(-\frac{\gamma}{2} \mathrm{e}^{-\frac{\gamma(t-\tau)}{2}}\right) \mathrm{d} \tau\right)^{2} \\
+\frac{2 B^{2}}{-\lambda_{k}} \sup _{\tau \in\left(t_{1}, t\right)}\|f(\cdot, \tau, y(\cdot, \tau))\|_{L^{2}(0,1)}^{2}\left(\int_{t_{1}}^{t} \mathrm{e}^{-\frac{\gamma(t-\tau)}{2}} \mathrm{~d} \tau\right)^{2} \leq \frac{8}{-\lambda_{k}} B^{2} \sup _{\tau \in\left(t_{1}, t\right)}\|f(y(\cdot, \tau))\|_{L^{2}(0,1)}^{2}\left(\frac{1}{b(\gamma)}+\frac{1}{\gamma^{2}}\right)
\end{gathered}
$$

Again, as in the proof of Theorem 1.2 in Section 3.4.5, but now with the initial condition as in (4.7), 4.11) at time $t=t_{1}$, we obtain that the last term on the right of (4.20) tends to zero along the relations (3.11), (3.15), (3.9), and (3.16a-b).

It remains to notice that, due to $(4.19),(1.4)$ and $(1.5)$ (similar to $(2.2 \mathrm{c})$ ),

$$
\sup _{\tau \in\left(t_{1}, t\right)}\|f(\cdot, \tau, y(\cdot, \tau))\|_{L^{2}(0,1)}^{2}
$$

is uniformly bounded for $t>0$ as in (3.15), and to use (3.18) and (3.12b)) to show that the first three terms in the right-hand side of (4.19) converge to zero as $\gamma \rightarrow \infty$ under the conditions (3.11), (3.15), (3.9), and (3.16a-b).

Combining this with the above evaluation of (4.12) yields that there is a pair of controls $(v, \gamma)$ and time $T>0$ described in Theorem 1.1 such that (1.7) holds. This completes the proof of Theorem 1.1.

\section{REFERENCES}

[1] A. Baciotti, Local Stabilizability of Nonlinear Control Systems. Ser. Adv. Math. Appl. Sci. 8 (1992).

[2] J.M. Ball and M. Slemrod, Feedback stabilization of semilinear control systems. Appl. Math. Opt. 5 (1979) 169-179.

[3] J.M. Ball and M. Slemrod, Nonharmonic Fourier series and the stabilization of distributed semi-linear control systems. Comm. Pure. Appl. Math. 32 (1979) 555-587.

[4] J.M. Ball, J.E. Mardsen and M. Slemrod, Controllability for distributed bilinear systems. SIAM J. Contr. Optim. (1982) $575-597$.

[5] M.E. Bradley, S. Lenhart and J. Yong, Bilinear optimal control of the velocity term in a Kirchhoff plate equation. J. Math. Anal. Appl. 238 (1999) 451-467.

[6] A. Chambolle and F. Santosa, Control of the wave equation by time-dependent coefficient. ESAIM: COCV 8 (2002) 375-392.

[7] L.A. Fernández, Controllability of some semilinear parabolic problems with multiplicative control, presented at the Fifth SIAM Conference on Control and its applications, held in San Diego, July 11-14 (2001).

[8] A.Y. Khapalov, Bilinear control for global controllability of the semilinear parabolic equations with superlinear terms, the Special volume "Control of Nonlinear Distributed Parameter Systems", dedicated to David Russell, G. Chen/I. Lasiecka/J. Zhou Eds., Marcel Dekker (2001) 139-155.

[9] A.Y. Khapalov, Global non-negative controllability of the semilinear parabolic equation governed by bilinear control. ESAIM: COCV 7 (2002) 269-283.

[10] A.Y. Khapalov, On bilinear controllability of the parabolic equation with the reaction-diffusion term satisfying Newton's Law. Special issue dedicated to the memory of J.-L. Lions. Computat. Appl. Math. 21 (2002) 1-23. 
[11] A.Y. Khapalov, Controllability of the semilinear parabolic equation governed by a multiplicative control in the reaction term: A qualitative approach. SIAM J. Control. Optim. 41 (2003) 1886-1900.

[12] A.Y. Khapalov, Bilinear controllability properties of a vibrating string with variable axial load and damping gain. Dynamics Cont. Discrete. Impulsive Systems 10 (2003) 721-743.

[13] A.Y. Khapalov, Controllability properties of a vibrating string with variable axial load. Discrete Control Dynamical Systems 11 (2004) 311-324.

[14] K. Kime, Simultaneous control of a rod equation and a simple Schrödinger equation. Syst. Control Lett. 24 (1995) $301-306$.

[15] S. Lenhart, Optimal control of convective-diffusive fluid problem. Math. Models Methods Appl. Sci. 5 (1995) 225-237.

[16] S. Lenhart and M. Liang, Bilinear optimal control for a wave equation with viscous damping. Houston J. Math. 26 (2000) $575-595$.

[17] M. Liang, Bilinear optimal control for a wave equation. Math. Models Methods Appl. Sci. 9 (1999) 45-68.

[18] S. Müller, Strong convergence and arbitrarily slow decay of energy for a class of bilinear control problems. J. Differ. Equ. 81 (1989) 50-67. 